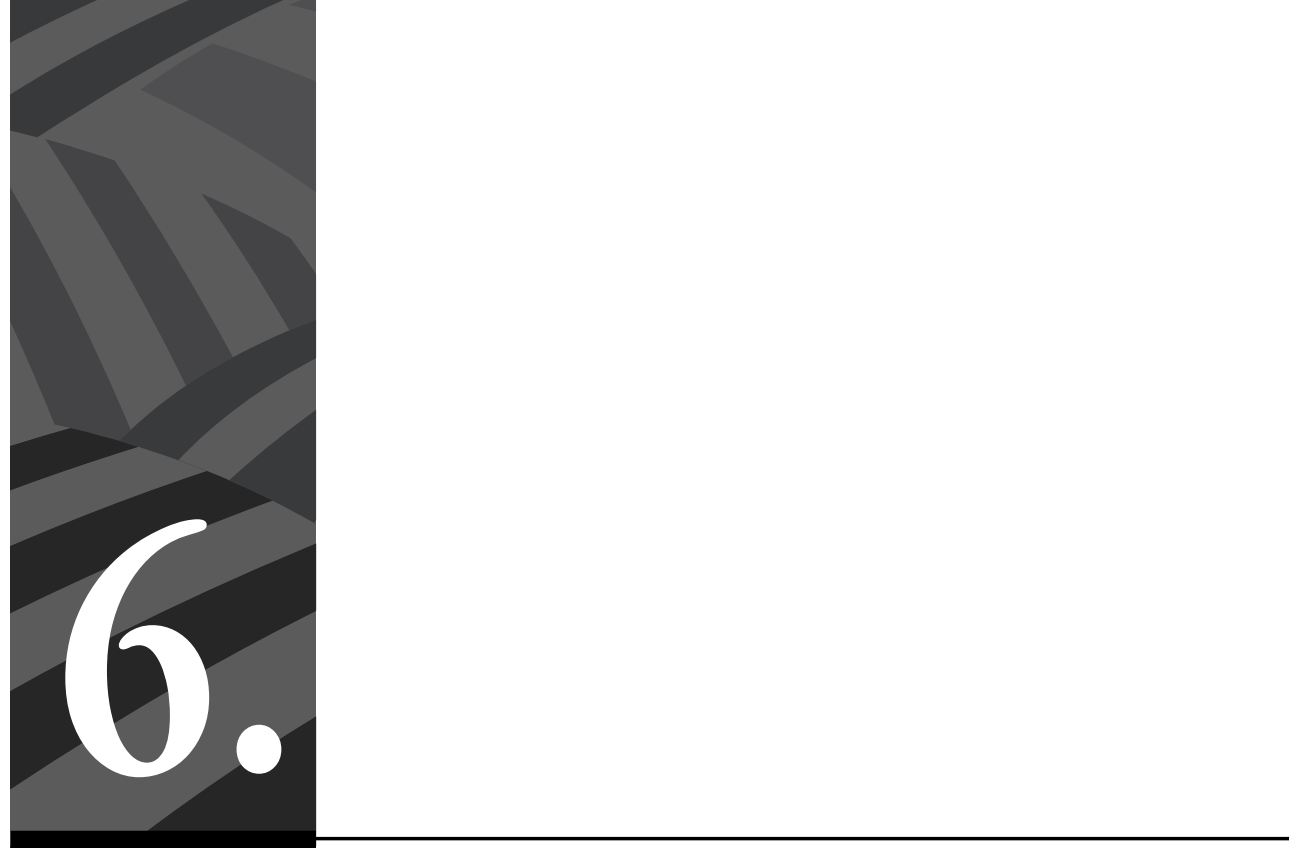

Notas acerca de la construcción curricular en la educación indígena 


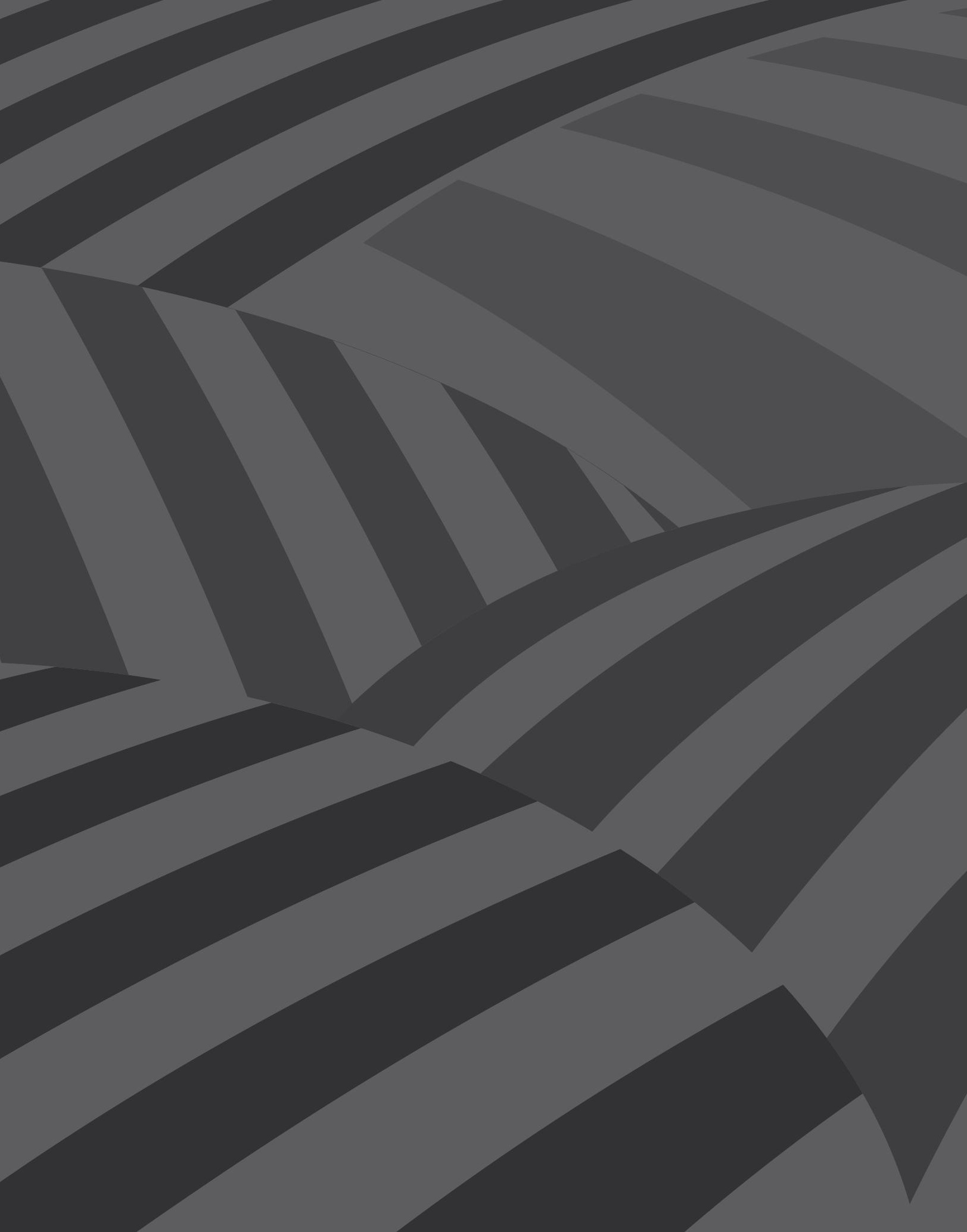




\title{
Notas acerca de la construcción curricular en la educación indígena
}

\author{
Por Santiago A. Gutiérrez Sánchez
}

Resumen: Este artículo presenta tres notas que nos permiten acercarnos a la construcción de planes de estudio y currículos de las instituciones educativas en territorios indígenas a partir de las experiencias colectivas y comunitarias de pueblos indígenas en el Departamento del Cauca (Colombia). El ensayo es producto de la experiencia de trabajo con docentes, organizaciones y comunidades indígenas durante el 2015 y 2016 en los municipios de Silvia y Jambaló. Estas notas son reflexiones personales y colectivas que presentan algunas tensiones pedagógicas que vive la educación escolar en territorios indígenas al suroccidente colombiano. Las cuales nos permiten acercarnos a los cambios que viven algunas instituciones educativas que atienden población indígena y afrontan el gran reto de construir currículos y planes de estudio en el marco de la educación propia y el Sistema Educativo Indígena Propio que adelantan las organizaciones indígenas.

Palabras clave: educación indígena, currículo, maestros comunitarios y movimiento étnico pedagógico

\section{Some notes regarding curriculum construction in indigenous education}

Abstract: This paper deals with three notes to approach curriculum construction in schools at indigenous territories. These notes are based on communal and collective experiences of the indigenous peoples of Departamento del Cauca (Colombia). This essay is the result of the work done with teachers, organizations and indigenous communities between 2015 and 2016 in the municipalities of Silvia and Jambaló. These notes are personal and collective reflections which unveil pedagogical tensions in school education at indigenous territories in the southwest of Colombia. This, in turn, allow us to understand the changes that some of these schools with indigenous students undergo, and the challenges they meet when designing curricula in the frame of their own education and that of the Sistema Educativo Indígena (Indigenous Educational System) promoted by indigenous organizations.

Keywords: indigenous education, curriculum, communal teachers, ethnic pedagogic movement.

* Antropólogo de la Pontificia Universidad Javeriana. Estudiante de Maestría en Educación de la Universidad de Antioquia. 
Cómo citar este artículo: Ávila Escobar, Nathalia y Montenegro Gómez, Gutiérrez Sánchez, Santiago A. (2017). Notas acerca de la construcción curricular en la educación indígena. Revista Controversia, 207-229.

Fecha de recepción: 2 de agosto del 2017

Fecha de aprobación: 18 de octubre del 2017

\section{Introducción}

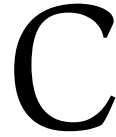
on la intención de proliferar escenarios de reflexión sobre la educación en Colombia, se presentan aquí algunas notas ${ }^{1}$ sobre la construcción de planes de estudio y currículo en territorios indígenas de la zona andina desde los aportes del campo de la antropología y la educación. El ensayo es producto de la experiencia de trabajo con organizaciones indígenas durante el 2015 y 2016, tiempo en el cual acompañé procesos de formación a niños, niñas, adolescentes y docentes indígenas en los municipios de Silvia y Jambaló, departamento del Cauca. Estas notas representan algunas tensiones o encrucijadas que vive la educación escolar en territorios indígenas al suroccidente de Colombia; por tanto, nos permiten acercarnos a los cambios que viven algunas instituciones educativas que atienden población indígena de los resguardos del municipio indígena de Jambaló, como también los retos que plantean los docentes al momento de construir planes de estudios.

El texto se divide en tres notas: la primera trata la relación en Colombia entre la escuela y los conocimientos indígenas desde los aportes académico o bibliográficos; la segunda nos acerca al lugar que tienen o cumplen los(as) docentes o maestros en las instituciones educativas en territorios indígenas; y la última nota presenta algunas reflexiones acerca de la construcción de proyectos educativos y planes de estudio en territorios indígenas en el Resguardo-Municipio Indígena de Jambaló (Cauca).

1 Notas: experiencias y fuentes documentales que se replantean a la luz de un tema o situación particular. 


\section{La escuela y los conocimientos indígenas: las tensiones del PEC}

Durante el período comprendido entre el siglo XVII y principios del XIX, se dieron en el mundo algunos avances sobre los conceptos de niño(a) y de educación. Así mismo, la disminución de la mortalidad infantil en el siglo XIX y la creciente industrialización de las sociedades “occidentales” o europeas llevaron a que se incrementara el fenómeno de la enseñanza primaria en el siglo xix y el de la secundaria en el Xx. La enseñanza se convirtió en el medio común de la mayoría de las aspiraciones nacionales: civilización, poder militar, desarrollo industrial, democracia, igualdad y construcción de una ciudadanía patriótica. No obstante, no se puede afirmar que estas tendencias de enseñanza hayan sido acontecimientos históricos únicos; por el contrario, los procesos históricos en los "países de desarrollo tardío” en tanto a la educación infantil no pueden verse como una simple reproducción de las reglas sociales y costumbres europeas (Levine y White, 1986, p. 68).

La historia de la educación en los pueblos indígenas de Latinoamérica está cruzada con los procesos de dominación y resistencia que se han presentado en el territorio y las poblaciones, entre los cuales es posible identificar por lo menos tres grandes momentos. El primer momento coincide con el proyecto de evangelización en el período de colonización (siglos XVII-XVIII). Posteriormente, durante el período republicano del siglo XIX se promueve desarrollar una especie de integracionismo escolar que da lugar a la institucionalización de las misiones como dispositivo educador en las regiones habitadas por indígenas y negros. Por último, encontramos la etapa de los derechos étnicos, en donde se da el reconocimiento de la educación para grupos étnicos y la cooptación mediante la institucionalización-oficialización (Rojas y Castillo, 2005). En el departamento del Cauca, Colombia, la presencia más significativa de misiones católicas se dio en la región de Tierradentro, donde las congregaciones de lazaristas y vicentinos administraron la educación de las poblaciones indígenas (Rojas y Castillo, 2005, p. 69). 
Las reformas que promovieron la modernización del sector educativo a partir de la década de los sesenta no afectaron el modelo de Iglesia Docente, ya que se concentraron en ampliar la cobertura en secundaria, la promoción de la educación superior pública y la centralización del financiamiento educativo. A partir de este momento, y durante el resto del siglo Xx, la educación y las políticas educativas fueron entendidas como estrategias de ascenso social y acceso a bienes materiales y simbólicos, así como de accesibilidad al mundo laboral (Rojas y Castillo, 2005).

En la región del suroccidente colombiano, el Consejo Regional Indígena del Cauca (CRIC) y otros procesos organizativos indígenas han impulsado desde hace más de treinta años propuestas educativas. Asimismo, durante la década de los noventa surgieron varias organizaciones indígenas zonales, como la Asociación de Cabildos Indígenas del Norte del Cauca (Acin) y el Consejo Territorial de Autoridades Indígenas del Oriente Caucano, con el propósito de implementar proyectos sociales y culturales encaminados a fortalecer la autonomía, la cultura y la unidad de los pueblos indígenas. Varias de estas organizaciones han impulsado la construcción de propuestas y experiencias educativas en diferentes comunidades, como por ejemplo el Proyecto Educativo Comunitario $(\mathrm{PEC})^{2}$, los proyectos escolares de investigación comunitaria, la Universidad Autónoma Indígena Intercultural (UAIIN) y el Sistema Educativo Indígena Propio (SEIP).

El PEC es una propuesta reconocida y adoptada por el Estado dentro de la normatividad educativa, la cual surgió en la década de los noventa (90`s) con la intención de permitir que las instituciones educativas oficiales ubicadas en territorios indígenas pudieran desarrollar un Proyecto Educativo Comunitario en vez del Proyecto Educativo Institucional (Bolaños et al., 2004, p. 71). Respecto a esto, Benavides (2013) da el ejemplo de la escuela de Ayanku'c o Agoyán del resguardo indígena de

2 Para profundizar sobre la participación de las organizaciones o asociaciones indígenas en los PEC ver: Rueda, Gómez y Martínez, 2003. 
Ambaló ${ }^{3}$ (Silvia, Cauca), la cual acogió desde muy temprano la construcción de escuelas comunitarias y, luego, la construcción del PEC; actualmente, la comunidad desarrolla una propuesta pedagógica que le permita recuperar la lengua materna y fortalecer la historia del pueblo ambalueño desde la Institución Educativa Técnica Ambaló.

La construcción del PEC plantea la articulación de los ámbitos educativos y administrativos de la educación con las necesidades, procedimientos y autoridades de las comunidades indígenas (Bolaños et al., 2004). Así, desde la propuesta de los cabildos indígenas nasa del municipio de Toribío ${ }^{4}$, el PEC revitaliza los saberes, actores y prácticas propias de los pueblos indígenas dentro del espacio educativo escolarizado, buscando cambiar lógicas y conocimientos que tradicionalmente se "imparten" en la escuela (CECidic, 2015). Por su lado, el Núcleo de Educación del Cabildo Indígena de Jambaló ha recorrido más de veinte años construyendo el PEC, planteando orientaciones para la edificación de instituciones, planes educativos, principios pedagógicos y planes de aula integrados (Cabildo Indígena de Jambaló, 2013).

Para el CRIC y la Mesa de Pueblos Indígenas (2007), el trabajo con el PEC realizado por parte del Núcleo de Educación es muy valioso, pues ha permitido romper hitos alrededor de la educación propia. Esto se observa en el aumento de la población estudiantil y el número de docentes contratados, así como también en la producción de material educativo propio y la incorporación de materias relevantes para el contexto sociocultural (nasa yuwe y cosmovisión, organización política y plan de vida, entre otras). Sin embargo, los autores aclaran que los criterios de evaluación de los estudiantes y los docentes son aspectos en donde se revela la incompatibilidad de la intervención del Estado y de las

3 Esta escuela se creó después de la recuperación de tierras que adelantó la comunidad del resguardo de Ambaló en 1983, acompañada por el CRIC.

4 Resguardo Indígena de San Francisco, Resguardo Indígena de Toribío y Resguardo Indígena de Tacueyó. 
iniciativas adelantadas por la comunidad (CRIC y la Mesa de Pueblos Indígenas, 2007).

Lo anterior pone de relieve que propuestas como las del PEC se encuentran en un campo de tensión y lucha. Para Vasco (2002), el proyecto de etnoeducación que plantearon el Gobierno y algunas organizaciones indígenas en la década de los ochenta ocultaba relaciones de poder, jerarquías de saberes y formas de dominación de una sociedad a otra. En este sentido, plantea:

Los currículos dobles, aditivos o yuxtapuestos, aunque comparen entre sí las distintas culturas, no se pueden constituir en la base de la confrontación [...] No se trata de presentar los elementos de una y otra cultura unos al lado de otros y en el mismo plano, sino de hacer conciencia explícita del significado económico, social y político de la propia cultura, de contrastar la base de pensamientos y conocimientos que la subyacen y fundamentan con las que corresponden a la otra cultura. (Vasco, 2002, p. 232)

Dicha tensión no solo se encuentra en los procedimientos para conformar instituciones educativas, sino también en los conocimientos y contenidos que se plantean desde la escuela/aula. Los conocimientos indígenas son producidos en contextos históricos y culturales específicos y no están conformados por una serie de reglas o procedimientos preestablecidos. Adicionalmente, los sistemas de conocimientos indígenas abarcan cosmovisiones, valores y prácticas culturales que se caracterizan por la transmisión oral y el aprendizaje a través de la experiencia y la práctica repetitiva (Breidlid, 2016).

Sin embargo, los contenidos, los espacios y la forma como es concebida la escuela hacen que estos conocimientos sean jerarquizados e instrumentalizados, pues se les da mayor centralidad y un carácter "universal” a los conocimientos "occidentales”, lo cual lleva a que los estudiantes indígenas se sientan relegados debido a que los conocimientos que traen de casa no son discutidos o valorados en el aula de clases (Breidlid, 2016). Esto pone de manifiesto que la tensión que ocurre en la 
conceptualización y construcción de la política etnoeducativa también se presenta en las instituciones educativas y en la construcción de proyectos pedagógicos; en este sentido, los PEC se muestran como un escenario clave en donde se encarna dicha tensión.

La educación propia o etnoeducación (escolarizada) vive varias ambivalencias debido a los procedimientos y políticas del Estado - como la selección docente, los contenidos o competencias de los planes de estudio y la evaluación-, así como a algunos imaginarios sociales que intervienen directamente en los actores educativos. Tal como afirman Rojas y Castillo (2005), esta tensión continúa con el paso del tiempo, ya que el reconocimiento o exposición de lo “cultural”, lo "indígena” y la "diferencia” en la escuela no pone en discusión los conocimientos "occidentales”, las lógicas de dominación/exclusión, los planes de inversión ni los espacios y métodos pedagógicos que aparecen como "universales”. Todo esto encubre la racialización e inferiorización de formas de ser y conocer de diferentes sociedades en la escuela. Para comprender lo anterior expondré, más adelante, el caso de la Institución Educativa Üus Kipni` Kiwe, del Resguardo Indígena de Jambaló (Cauca).

\section{Maestros(as), docentes, dinamizadores(as): ¿movimiento pedagógico étnico?}

Desde su creación en 1971, dentro de la plataforma de lucha del CRIC aparece la formación o nombramiento de maestros indígenas, considerados como agentes de poder de cambio en las comunidades (Bolaños, 2009). Esto implica, por tanto, la necesidad de preparar un tipo de maestro diferente al de las escuelas normalistas o universidades. El surgimiento de los “maestros(as) comunitarios(as)" constituye un importante acontecimiento del movimiento indígena - en especial en las escuelas comunitarias autogestionadas por las comunidades-, ya que contiene elementos constitutivos que provienen de la manera como las organizaciones y comunidades concibieron la educación y el oficio del docente en el marco de la lucha política y la vida comunitaria (Castillo, 2014, p.51). 
Según Rojas y Castillo (2005), el Decreto 1142 de 1978 se constituye como referente de un movimiento pedagógico étnico, ya que introduce en la literatura oficial el concepto de "etnoeducación” o "educación indígena”, a la vez que respalda una constante lucha por participar en la formulación de las políticas educativas nacionales ${ }^{5}$. Ese mismo año, en el departamento del Cauca se crea el Programa de Educación Bilingüe Intercultural (Pebi) del CRIC, el cual empieza a trabajar diferentes aspectos sobre educación, escolarización y profesionalización docente. Adicionalmente, con la intención de avanzar en el reconocimiento estatal de los(as) maestros(as) indígenas, entre 1988 y 1999 el CRIC se asocia con algunas Escuelas Normales Superiores (ENS) y emprende programas de formación para que sus maestros comunitarios obtengan su titulación como bachiller pedagógico (Castillo, 2014).

La Constitución de1991 recoge, por lo menos en el papel, varias reivindicaciones del movimiento indígena. En concordancia con esto, la normatividad educativa posconstituyente deja ver un cambio en la política etnoeducativa del Estado; el Decreto 804 de 1994, por ejemplo, manifiesta una prioridad en la dimensión pedagógica de la etnoeducación mediante el establecimiento de componentes de la normatividad educativa ordinaria como: diseño curricular, investigación, material educativo, asesoría, seguimiento y evaluación. Esta normatividad da existencia legal y oficial a los etnoeducadores y las licenciaturas en etnoeducación (Castillo, 2014).

No obstante, a pesar de que los derechos de los pueblos indígenas, reconocidos a finales del siglo xx, manifestaron la obligatoriedad de adecuar la política e institucionalidad educativa a las necesidades, exigencias y saberes indígenas/afros, el cambio en la política educativa para grupos étnicos y el reconocimiento de su derecho a la educación no se ha dado de manera natural, uniforme y planificada. Lo anterior se debe,

5 Resulta pertinente aclarar que este movimiento no fue impuesto, ya que respondió a los procesos/necesidades de los diferentes pueblos indígenas. 
entre otras cosas, a que no se establecieron procedimientos claros, con lo cual se han saltado procesos sociales y culturales necesarios para su implementación (Rojas, 2006).

Asimismo, las leyes de educación para grupos étnicos y las acciones institucionales en el campo de la etnoeducación se enfrentan a una condición de marginalidad que define los límites y alcances de su acción; en especial tras la reforma educativa implementada mediante la Ley 715 de 2001 (Rojas y Castillo, 2005). Todo esto ha generado que las acciones institucionales de los programas de etnoeducación estén insertas en las lógicas de funcionamiento burocrático, bajo criterios como los de modernización y racionalización de recursos (Rojas y Castillo, 2005).

El caso de las organizaciones indígenas del departamento del Cauca es enriquecedor, ya que generaron procesos sociales y comunitarios que permitieron avanzar en la construcción de la educación propia incluso en medio de las reformas educativas que implementaba el Estado. Un ejemplo de esto tiene que ver con los convenios realizados entre el Ministerio de Educación Nacional (MEN), la Secretaría de Educación Departamental (SED) y el CRIC para la contratación docente ${ }^{6}$, pues con estos lograron diversificar las relaciones que tenía el(la) docente con la comunidad.

A partir de estos convenios empezaron a coexistir en las instituciones educativas de los territorios indígenas del Cauca docentes contratados por la SED (provisionales), por el MEN (los de propiedad o planta) y por la organización indígena regional (oferentes). La intención de las organizaciones indígenas era que los(as) profesores(as) oferentes empezaran a cuestionar los proyectos pedagógicos que se habían desarrollado hasta ahora en las instituciones educativas en donde iban trabajar (Gutiérrez, 2013). Sin embargo, esto generó divisiones y tensiones entre los docentes,

6 Entre 1997 y 2005, mediante diferentes acciones el CRIC le exigió al Estado reconocer y operativizar el (SEIP), lo que resultó en una serie de convenios de contratación docente para territorios indígenas entre el PEBI, la SED y el MEN (CRIC, 2011). 
pues adicionalmente tenían cargas laborales y remuneración económica diferentes.

Como puede verse, las organizaciones indígenas han dado pasos importantes tanto en la construcción de las políticas etnoeducativas ${ }^{7}$, como también en la consolidación de experiencias pedagógicas bilingües y comunitarias. En este sentido, las organizaciones plantean el Sistema Educativo Indígena Propio (SEIP) como el modelo pedagógico, político y administrativo de la implementación de la educación pública en territorios indígenas. Un elemento central del (SEIP) es su componente pedagógico, en donde el dinamizador es una pieza o sujeto fundamental para la transformación del modelo educativo oficial al desplegar diferentes estrategias y espacios para consolidar un campo intelectual y narrativo de la pedagogía de la educación propia, en términos de Echeverri (2015). Es tan importante la figura del docente para estas organizaciones indígenas, que el CRIC crea la Licenciatura en Pedagogía Comunitaria en la Universidad Autónoma Indígena e Intercultural -UAIIN-, la cual cuenta con diferentes programas para dar respuesta a las necesidades de las comunidades.

Sin embargo, a pesar de estos avances, también han aparecido dificultades en el camino. Según Tatay (2008), por ejemplo, en algunos casos el Estado visibiliza su postura sobre lo indígena como "incapaz” o "atrasado": esto sucede sobre todo a través de las lógicas e imaginarios que portan quienes operativizan la educación en territorios indígenas, así como también a partir de las posturas de algunos padres de familia, comuneros y comuneras que ven la propuesta educativa del Estado como más legitima y con mayor posibilidad de "hacer" sujetos "exitosos".

7 Los decretos y resoluciones concertados y elaborados desde el seno de las organizaciones indígenas han sido varios. Podemos citar dos: Decreto Transitorio 2500 del 2010 y Decreto 1953 del 2014. 
La lógica comunitaria de la propuesta educativa de las comunidades y organizaciones indígenas encuentra su mayor desafío al enfrentarse a la lógica institucional que habita en las estructuras de seguimiento y control de los espacios educativos, las cuales se distinguen por su carácter homogeneizador y su ojo vigilante. Igualmente, las visiones y procederes de los actores educativos locales, como padres de familia, docentes y directores de núcleo, suponen también un importante desafío para esta propuesta educativa comunitaria. (Tatay, 2008)

En suma, considero que la existencia de un movimiento pedagógico étnico representa un cambio en el saber-hacer educativo, pues ve en la escuela y en el quehacer docente una forma de mejorar la calidad de vida de las personas mediante la promoción de diversas clases de conocimiento, de participación y de convivencia. Esto, como vimos, se refleja en las diferentes experiencias y propuestas realizadas desde las organizaciones indígenas, por ejemplo la formación de maestros, los proyectos pedagógicos y la transformación del saber específico y quehacer pedagógico (maestros y maestras comunitarios). La figura del(la) dinamizador resulta central para concretar estas propuestas en medio de las tensiones que se dan entre la educación propia y la normatividad etnoeducativa.

La organización de este movimiento étnico pedagógico no es uniforme, a pesar de haberse contemplado desde las organizaciones indígenas (CRIC, Acin, Cotaindoc, etc.); por el contrario, es cada vez mayor la diversificación de docentes que participan en los espacios de formación adelantados por las organizaciones indígenas y que laboran en instituciones educativas dentro de territorios indígenas. En otras palabras, el movimiento pedagógico étnico se estructuró como campo pedagógico y narrativo dentro de las organizaciones indígenas, mas no se edificó como un gremio del quehacer docente autónomo. 


\section{Encrucijadas educativas. El caso de la Institución Educativa "Ūus Ki'pni Kiwe"}

Para ilustrar y acercar las anteriores notas a lo que sucede en la escuela, voy a presentar el trabajo que he adelantado junto con docentes indígenas y no indígenas, autoridades indígenas y niños, niñas y adolescentes del Resguardo Indígena de Jambaló, en el departamento del Cauca; especialmente el trabajo realizado durante el 2016 en la Institución Educativa Agrocultural “Ūus Ki’pni Kiwe” en la zona alta del resguardo, en la vereda Paletón.

En el Resguardo-Municipio de Jambaló, la idea de un currículo o escuela propia surgió de líderes y docentes indígenas a través de la escuela comunitaria impulsada en Vitoyó. No obstante, la construcción del PEC de manera estructural inició a mediados de los años noventa con el acompañamiento de la Fundación Caminos de Identidad (Fucai). Entre 1995 y 1996, esta fundación, la Secretaría de Educación Departamental y el Cric desarrollaron talleres sobre PEC y PEI con docentes y comunidades. A partir de entonces, se continuó realizando con los y las docentes el trabajo de sensibilización del PEC y la propuesta de formación por núcleos temáticos. Además, durante la primera década del siglo XXI, el Cabildo Indígena de Jambaló, la Alcaldía Municipal y la Acin implementaron talleres sobre educación y currículo propio (Cabildo Indígena de Jambaló, 2013).

El PEC del Resguardo Indígena de Jambaló integra diferentes áreas del conocimiento en seis grandes núcleos temáticos: Comunicación y lenguaje; Territorio, naturaleza y sociedad; Producción y economía del desarrollo para el bienestar; Participación política y organización social de los pueblos indígenas y no indígenas; y Ser persona: nas nasa, nam misak y/o mestizo. Estos núcleos temáticos se trabajan a través de los planes de estudios y de aula que se elaboran mediante "Unidades de aprendizaje” en primaria y "Ejes de aprendizaje” en secundaria (Cabildo Indígena de Jambaló, 2013). 
La escuela de la vereda Paletón fue creada aproximadamente entre 1969 y 1970 e inició en la casa de familia del señor Mario Cuetía. Para finales de los años ochenta, los estudiantes de los diferentes grados eran atendidos por una sola docente. En 1995 había tres docentes que trabajaban con la modalidad de "escuela nueva" con los grados de primero, segundo, tercero, cuarto y quinto. Actualmente, el Centro Educativo Paletón se convirtió en la sexta Institución Educativa del resguardo-municipio, denominada Institución Educativa Agrocultural “Ūus Ki’pni Kiwe”, y ofrece la formación agrocultural desde primaria hasta grado décimo (secundaria). El plan de estudios de bachillerato parte de los lineamientos y planteamientos del PEC del resguardo-municipio de Jambaló, por lo cual acoge el trabajo por núcleos temáticos, unidades de aprendizaje y ejes transversales. En la actualidad, la institución cuenta con un directivo y diez docentes, cinco en primaria y cinco en secundaria; la mayoría de estos son docentes provisionales o contratados, solo dos son de propiedad o de planta, contratados directamente por las entidades territoriales.

Uno de los primeros ejercicios que realizamos fue retomar las necesidades que la comunidad y el cuerpo docente habían identificado con una matriz DOFA en el 2002, y las analizamos de acuerdo al contexto que estaba viviendo la comunidad en el año 2016. Este ejercicio permitió ver que debido a la coyuntura nacional habían cambiado algunas situaciones que antes se presentaban con más fuerza; por ejemplo, el conflicto armado y la presencia de grupos armados que, aunque siguen latentes, se presentan de otra manera tras los acuerdos de paz con las FARC. De igual manera, los programas sociales y estatales ${ }^{8}$ aparecieron como un aspecto que se debe tener en cuenta y analizar, ya que interviene de una u otra manera en el ámbito educativo y en las lógicas comunitarias $^{9}$. Durante estos ejercicios, los(as) docentes tanto de primaria como

8 Algunos programas estatales presentes en el resguardo son: De Cero a Siempre, ICBF, Más Familias, Programa de Alimentación Escolar.

9 Las lógicas y prácticas comunitarias son la minga, las asambleas, el trabajo colaborativo y colectivo, las prácticas culturales, entre otras. 
de secundaria identificaron o reconocieron también diferentes problemáticas o necesidades que vive la comunidad, en especial los niños, niñas y adolescentes escolarizados ${ }^{10}$.

Desde este momento, empezaron a surgir discusiones sobre los "límites” entre la educación formal y no formal: ¿hasta dónde llega el rol de la escuela y del/la docente frente a las necesidades o problemáticas identificadas? y ¿cómo los docentes y los planes de estudios tienen en cuenta los cambios sociales, económicos y culturales que vive la comunidad? Intentando responder estas preguntas, algunos docentes afirmaron que los programas sociales influyen en el desarrollo cognitivo y de personalidad de los niños y niñas indígenas que entran a la escuela. En este sentido, se aseguró, por ejemplo, que los programas que atienden a la primera infancia no tienen en cuenta el uso de la lengua, lo que ha generado la pérdida de la lengua y su conciencia entre niños y niñas. También se discutió sobre algunos valores o actitudes sociales y culturales que los niños y niñas deben haber adquirido en su familia antes de entrar a la escuela y que no deben provenir únicamente de la relación docente-estudiante, como el respeto por las demás personas y las prácticas de higiene.

En discusiones alternas y conversaciones cotidianas, algunos docentes exponían que la revitalización de la lengua materna (nasa yuwe) se debería hacer en la familia y en programas sociales que atienden a la primera infancia. Lo anterior no quiere decir que el personal docente no esté de acuerdo con las clases de cosmovisión y lengua nasa yuwe que se dan en primaria y secundaria, pero sí refleja una disposición del uso y concienciación de la lengua materna que tienen algunos docentes tanto en los planes de estudio como en el aula. En contraste, algunos

10 Algunas de las problemáticas identificadas fueron: el consumo de sustancias psicoactivas y alcohol, los cultivos ilícitos y el narcotráfico, la presencia de grupos armados, la pérdida de la identidad y los conocimientos ancestrales indígenas como la lengua, los tejidos y los rituales. 
docentes indígenas reconocen la importancia de la oralidad en la construcción y transmisión de conocimiento en el pueblo nasa, como también en el proceso de aprendizaje-enseñanza.

La comunidad y los docentes de esta institución educativa desde hace varios años tomaron la decisión de orientar el desarrollo del modelo educativo hacia la revitalización cultural, por lo cual plantearon un énfasis "agrocultural". Los líderes, lideresas y docentes manifestaron que buscan hacer de esta institución educativa una propuesta de educación alternativa, bilingüe, agrícola y cultural, "pionera en el resguardo". Si bien la orientación de la comunidad es tomar lo agrocultural como actividades culturalmente específicas, los(as) docentes (indígenas y no indígenas) consideran que la modalidad agrocultural consiste en realizar un diálogo y contraste entre los saberes propios/locales con los conocimientos técnicos agroambientales, pues de esta forma se puede indagar sobre los saberes y prácticas socioculturales propios y locales realizando una lectura alrededor de la influencia de los modelos económicos y las lógicas de comercialización y transformación de los productos. Así, para los docentes lo agrocultural no es un campo o área científica determinado, sino que son actividades específicas que articulan, contrastan y ponen en práctica conocimientos tradicionales de los pueblos indígenas y campesinos en relación con el territorio (producción, sustento económico, convivencia, simbolismo, prácticas socioculturales) y las áreas del conocimiento (Institución Educativa Académica Paletón, 2016).

En este sentido, lo agrocultural no solo se refiere a acciones de siembra y cosecha de alimentos, sino a diferentes saberes, prácticas, valores y relaciones entre el hombre y la naturaleza que permiten mantener la armonía y equilibrio en el territorio; esto lo consiguen profundizando en actividades y necesidades específicas de acuerdo con el interés y las capacidades dela comunidad educativa. Algunos escenarios comunitarios en donde se socializan y ponen en práctica de manera integral los conocimientos agroculturales son las mingas (trabajo colectivo), el trueque 
y los rituales sagrados (Sakheluu, Sek Buy, entre otros), escenarios en donde participan docentes en el marco de su quehacer pedagógico.

Los(as) docentes de primaria de la I.E. Uus Ki'pni Kiwe vienen implementando hace más de tres años la metodología del trabajo integrado de las áreas que propone el PEC de Jambaló, a partir de las Unidades de aprendizaje o Ejes temáticos; sin embargo, los(as) docentes de secundaria no están familiarizados con esta propuesta. En bachillerato o secundaria, los docentes trabajan a través de planes de estudios y contenidos establecidos previamente, ya sea por el docente o por la normatividad del Ministerio de Educación.

La reflexión sobre el trabajo por unidades temáticas y la construcción de conocimientos en los procesos de enseñanza-aprendizaje escolares por parte de los docentes de primaria son bastante enriquecedoras. Las unidades permiten que los(as) docentes vean diferentes áreas del conocimiento o saber en una jornada escolar. Así mismo, promueven articular la escuela con espacios y actores no escolares, tal como sucede con el trabajo en las huertas familiares y con la vinculación de los mayores y mayoras a los procesos de enseñanza-aprendizaje. Una de las conclusiones manifestadas por los(as) docentes es la ineficacia de los horarios y la distribución por áreas, ya que trabajar las Unidades temáticas les ha permitido trabajar los conocimientos y contenidos exigidos por el MEN de una manera más espontánea y creativa a partir de situaciones cotidianas y familiares para los niños y niñas.

Los contenidos alrededor de lo agrocultural abarcan tanto los saberes propios y locales como los conocimientos científicos y sobre modelos económicos imperantes, con lo cual se busca integrar estos saberes y conocimientos. Así, por ejemplo, se espera que el estudiante profundice sobre los calendarios y alimentos propios, la forma de siembra de los mayores y los rituales de armonización y equilibrio, pero también sobre las principales consecuencias de la contaminación y degradación de los 
suelos y sobre las formas industriales de producción, comercialización y explotación de los recursos naturales.

Un ejemplo de la transversalidad en los procesos de enseñanza-aprendizaje agrocultural es la lengua materna (nasa yuwe), ya que para los docentes las formas de trasmisión y reproducción de la lengua contemplan necesariamente espacios y acciones relacionados con el trabajo de la tierra, el trabajo comunitario, el conocimiento de los sitios sagrados y los animales del entorno, comer los alimentos propios, entre otros casos sociales y culturales. Lo que contrasta con el saber especifico, ya que este ejercicio no se realiza con las áreas del conocimiento "occidental" como matemáticas, química, física, español.

Por otro lado, respecto a las situaciones problemáticas reconocidas en el plan de estudios, los(as) docentes identificaron posibles escenarios sociales y educativos potenciales para afrontarlas y superarlas. Así, para lograr este objetivo se resalta la necesidad de realizar los procesos de formación por fuera del aula, a través, por ejemplo, de actividades cotidianas o comunitarias (truque, asambleas, rituales). Estas reflexiones muestran la tensión existente en la construcción de los planes de estudios, ya que la importancia de fijar límites o fronteras entre el saber docente (saber especializado) y los conocimientos/valores indígenas se sobrepone sobre los nuevos escenarios y metodologías aplicadas por los docentes para trabajar la integralidad o transversalidad del currículo.

Es necesario reconocer los avances y las reflexiones que han alcanzado las organizaciones y comunidades indígenas en torno a la educación propia. Por eso, tras haber caminado sobre sus huellas, considero que para abordar la construcción de planes de estudios o currículos en instituciones educativas en territorios indígenas es necesario comprender la relación que hay entre la escuela y los conocimientos indígenas. Hay también que preguntarse por el lugar que han tenido históricamente niños, niñas, padres y madres de familia, comunidades y docentes en esta relación, ya que las tensiones y contrapunteos no solo se presentan 
en la normatividad y conceptualización de la educación, sino también en los diferentes espacios y procedimientos escolares, como por ejemplo en el aula, en la elaboración de planes de estudio, en los proyectos educativos y en el quehacer pedagógico. Estas tensiones no se presentan siempre de manera explícita, pues en ocasiones se las considera como algo "políticamente incorrecto" o moralmente desaprobado; sin embargo, aparecen en las discusiones cotidianas sobre la planeación educativa y el quehacer docente.

Considero que para afrontar dichas tensiones es necesario acercarnos y entender detenidamente la segunda nota, es decir el lugar y el quehacer de los(as) docentes en las instituciones educativas que se encuentran en territorios indígenas. Si bien los docentes y maestros comunitarios son sujetos centrales en la reflexión pedagógica dentro del (SEIP), las organizaciones indígenas no plantean aún la concepción de un movimiento pedagógico étnico. No obstante, la idea de un movimiento de ese tipo nos permitirá recuperar y reinterpretar la historia de los docentes indígenas y comunitarios que han adelantado procesos de enseñanza-aprendizaje poniendo en cuestión contenidos, pensamientos y estereotipos establecidos por la sociedad mayoritaria. Todo esto nos recuerda la importante relación histórica que hay entre conocimientos y sociedades dentro de la planeación y construcción curricular que hacen los docentes, lo cual nos permite reconocer el campo conceptual y narrativo de la pedagogía propia de la educación para pueblos indígenas.

\section{Referencias}

Benavides, Angie Linda (2013). Experiencia educativa resguardo indígena de Ambaló. Escuela Ayanku'c. En Sandra Patricia Guido (Coord.). Experiencias de educación indígena en Colombia: entre prácticas pedagógicas y políticas para la educación de grupos étnicos (pp. 57-85). Bogotá: Universidad Pedagógica Nacional. IUP.

Bolaños, Graciela (1998). Educación comunitaria: una fuerza que da sentido a la vida de los pueblos indígenas del Cauca. Nómadas, (9), 121-127. 
Bolaños, Graciela (2009). Desde la escuela y hasta la universidad: para un buen y mejor gobierno. En L. E. López (Ed.). Interculturalidad, educación y ciudadanía. Perspectivas latinoamericanas (pp. 355-377). Bolivia: Fundproeib Andes, Editorial Plural.

Bolaño, Graciela; Ramos, Abelardo; Rappaport, Joanne; y Miñana, Carlos (2004). ¿Qué pasaría si la escuela...? 30 años de construcción de una propuesta de educación propia. Programa de Educación Bilingüe e Intercultural. Popayán: Cric.

Breidlid, Anders (2016). Educación, conocimientos indígenas y desarrollo en el sur global. Cuestionando los conocimientos para un futuro sostenible. Buenos Aires: Clacso.

Cabildo Indígena de Jambaló (2013). Proyecto Educativo Comunitario. Jambaló: Núcleo de Educación. Cauca.

Castellanos, Marcela y Caviedes, Mauricio (2007). La educación en los pueblos indígenas de Colombia. En Indígenas sin Derechos: Situación de los derechos humanos de los pueblos indígenas. Bogotá: Cecoin. OIA.

Castillo, Elizabeth (2014). Pedagogía comunitaria y maestros comunitarios indígenas. Revista Integra Educativa, VII(1), 45-60.

Caviedes, Mauricio (2016). El debate sobre la educación escolar indígena: posibilidades desde el análisis antropológico. En Mauricio Caviedes y Maritza Díaz (Eds.). Infancia y Educación. Análisis desde la antropología (pp.219232). Bogotá: Pontificia Universidad Javeriana.

CECIDIC. (2015). Guía de Investigación. Guía para estructurar y desarrollar procesos investigativos en el Territorio Nasa de Toribío, Tacueyó y San Francisco. Texto de creación colectiva - Investigación CECIDIC. Toribío, Cauca (Colombia).

CRIC, Mesa de Pueblos Indígenas y DESC (2007). Indígenas Sin Derechos. Situación de los derechos humanos de los pueblos indígenas. Bogotá: Cecoin, Organización Indígena de Antioquia.

CRIC (2011). Sistema Educativo Indígena Propio. Primer Documento de Trabajo. Popayán: Cric-Pebi.

Echeverri, Jesús Alberto (2015). Desplazamiento y efectos en la formación de un campo conceptual y narrativo de la pedagogía en Colombia (19892010). En: J. A. Echeverri (Ed.). Paradigmas y conceptos en educación y pedagogía (pp. 147-198). Bogotá: Siglo del Hombre. 
Gros, Christian y Morales, Trino (2009). ¡A mí no me manda nadie! Historia de vida de Trino Morales. Bogotá: Icanh.

Gutiérrez, Santiago (2013). Los caminos de la educación propia: una aproximación al Programa de Educación del Consejo Territorial de Autoridades Indígenas del Oriente Caucano - Cotaindoc - (tesis pregrado de antropología). Pontificia Universidad Javeriana, Bogotá.

Institución Educativa Académica Paletón. (2016). Plan de Estudios de la Institución Educativa Académica Agrocultural Üus Ki’́pni Kiwe. Documento no publicado. Núcleo de Educación del Cabildo Indígena de Jambaló. Jambaló, Cauca (Colombia).

Levine, Robert (1986). El hecho humano. La base cultural del desarrollo educativo. Madrid: Visor/MEC.

Miñana, Carlos (2003). Educación, etnoeducación y neoliberalismo: el punto de vista de los "nativos" (neoliberales). Bogotá: Universidad Nacional de Colombia, Programa RED. Recuperado de: http://www.humanas.unal.edu. co/red/files/6312/7248/4191/Artculos-etnoeducacion.pdf

Pancho, Avelina (2007). Una decisión comunitaria para avanzar en la autonomía. Çxatu'çe Revista de Etnoeducación. Popayán: Cric.

Rodríguez, Lidia Mercedes (2014). Historia de la educación latinoamericana: aportes para el debate. En Myriam Southwell y Nicolás Arata (Comps.), Ideas en la educación latinoamericana: un balance historiográfico (pp. 6577). Buenos Aires: Unipe Editorial Universitaria.

Rojas, Axel y Castillo, Elizabeth (2005). Educar a los otros. Estado, política educativa y diferencia cultural en Colombia. Popayán: Editorial Universidad del Cauca.

Rojas, Biviany (2006). Los procesos de etnoeducación: de las reivindicaciones y la ley al ejercicio de los derechos. En O. A. Garzón (Comp.). Educación, Escuela y Territorio en la Amazonia Colombiana (pp. 319-342). Bogotá: Fundación Gaia-Amazonas.

Rueda, Álvaro; Gómez, Consuelo y Martínez, Adán (2003). Proyectos educativos comunitarios en pueblos indígenas. Colombia: Fucai. Cordaid. 
Tatay, Libia (2008). La "educación propia" en territorios indígenas caucanos: escenarios de hegemonía y resistencia (tesis de maestría en Ciencias Sociales con mención en Antropología). Flacso, Ecuador.

Vasco, Luis Guillermo (2002). Entre selva y páramo, viviendo y pensando la lucha india. Bogotá: Icanh. 